\title{
UNA ALTERNATIVA PARA LA FORMACIÓN VIAL
}

\author{
Carolina Belén Tencio Blanco \\ Estudiante de la Maestría en Planificación Curricular \\ Universidad de Costa Rica \\ San José, Costa Rica
}

Recibido: 27-XI-2007 • Aceptado 11-III-2008 • Corregido 15-IV-2008

\begin{abstract}
Resumen: Este artículo muestra de manera general la problemática que actualmente se vive a nivel mundial, y que ha venido aumentado año tras año en la sociedad costarricense, con cifras alarmantes donde la edad, el sexo, el credo, status social no escapan a tal situación, y que afecta las diferentes áreas sociales que velan por el bienestar de todos, una problemática que desencadena un sin fin de consecuencias fatales: los accidentes de tránsito. Esta epidemia como la llama la OMS (Organización Mundial de la Salud), ha sido desde ya hace décadas una enfermedad silenciosa, donde no se encuentran soluciones eficaces que acaben con tantas secuelas que deja a su paso, a pesar del esfuerzo de muchas instituciones del país. Es así como se pretende desarrollar una iniciativa desde una perspectiva que oriente la búsqueda de soluciones, desde las raíces de nuestra cultura, por medio de una formación integral continua desde edades tempranas. Esta tarea sin lugar a dudas le corresponde a todos los que somos partícipes, pero la visión y misión de formar ciudadanos concientes de sus derechos, sin olvidar sus deberes en armonía con lo que le rodea desde principios que demuestran una sociedad más responsable y segura, le corresponde al sistema educativo costarricense, en conjunto con las diversas entidades que de una u otra forma son parte de esta situación. Esto con el objetivo de encontrar en la educación continua y sistemática, una herramienta efectiva que a mediano o largo plazo logre disminuir las estadisticas de muertes en carretera, por la negligencia o error humano, causa de mayor incidencia en los accidentes de tránsito, por medio de una formación en y para una cultura vial, cuya política e ideología sea respetar la vida en la búsqueda del bienestar común.
\end{abstract}

\section{Introducción}

El siglo XXI se caracteriza por tener cambios significantes y continuos. Esto demanda que el ser humano tome conciencia de lo que vive día con día, para lograr no solo adaptarse, sino también para modificar las situaciones que puedan estar ejerciendo efectos negativos para sí y la sociedad.

Es así como la seguridad vial se ha convertido no sólo en un problema nacional, sino también a nivel mundial, ya que cada año mueren cientos de personas alrededor del mundo por la falta de cultura vial.

Así lo afirma la OMS (Organización Mundial de la Salud), al considerar los accidentes de tránsito un problema de salud, además advierte en su informe global sobre la incidencia del tráfico del 2004, que para el año 2020, los accidentes de tránsito serán la tercera causa de morbilidad y muerte (ahora son la novena en el planeta), sólo superada por las patologías relacionadas con el corazón y las enfermedades mentales (OMS, 2004).

En Costa Rica, la Ministra de transportes González (2007), "asegura que el 
Palabras clave: Accidentes de tránsito, educación, cultur, formación integral, currículo.

\begin{abstract}
This article shows, in a general manner, the problems that are currently felt worldwide and that have been increasing year after year in the Costa Rican society, for which figures are alarming and regardless of age, sex, religion, social status, affect different social areas which look after everyone's wellbeing. These problems that lead to endless fatal consequences: car accidents. This "outbreak" as the WHO (World Health Organization) calls them, has been for decades a silent disease, for which solutions are hard to find, solutions that will put an end to many consequences it leaves on its wake, regardless of the efforts of many governmental institutions. Thus, the development of an initiative is intended in this work, from a perspective that will lead to the search for solutions. These solutions must come from the root of our culture, through continuous, integral education from an early age. The task, without a doubt, corresponds to all who are part of the problem, but the vision and mission to educate citizens aware of their rights, without overlooking their duties in harmony with their surroundings, based on principles that show a more responsible and safe society, corresponds to the Costa Rican Educational System, along with several entities that are part this situation. This has the objective of finding in continuous and systematic education, an effective tool that in the long term will decrease the statistic figures of deaths in the street, due to negligence or human error-cause of the highest incidence of traffic accidents. This could be possible through education in and for traffic culture, with the policies and the ideology to respect life, in search of the common good.
\end{abstract}

Key words: Traffic accidents, education, traffic culture, integral education, curriculum. país tiene una cultura vial que no toma en cuenta ni la vida, ni la ley", y explica que hay 66 familias que han sufrido la pérdida de un ser querido, sólo en lo que va del año 2007, 18 más que el año anterior en el mismo período.

Es por esto que el tema educación vial es una responsabilidad que compete a toda una sociedad, por lo que áreas como salud y educación deben estar presentes.

La seguridad vial es parte de nuestro diario vivir, porque de alguna manera nos vemos afectados positiva o negativamente según lo que acontece.

Debemos tomar no sólo conciencia del problema que nos aqueja, sino también de acciones para disminuir los accidentes de tránsito y así reducir las muertes en carretera.

En este artículo se pretende informar a la población de una realidad latente en nuestra sociedad, ya que es muy evidente que existe una problemática de educación vial que prevalece, donde se observa un aumento en pérdidas humanas por esta causa, a pesar de campañas a nivel nacional, programas de capacitación a docentes, publicidad radial, televisiva y vallas publicitarias, creación de libros para preescolar, primaria y secundaria con temas alusivos, en la búsqueda de una concientización.

Los programas que se desarrollan en la educación general básica propuesta por el Ministerio de Educación Pública (MEP), no contemplan una continua educación vial. Es una temática que se menciona en programas de preescolar y Estudios Sociales del I ciclo y luego en undécimo año, pero el proceso de formación del futuro ciudadano no está incluida.

Es por esto que el propósito será demostrar la necesidad de desarrollar un programa sobre educación vial, que se incorpore en todas las escuelas del país y que el mismo modifique la cultura vial que se evidencia en nuestras calles y estadísticas, logrando que cada peatón y conductor sea responsable no solo de su vida, sino también de la de los demás. 
La educación vial por lo tanto, debe ser un proceso continuo desde el primer ciclo, hasta la educación diversificada, para que sea efectiva, ya que estaría dentro de un tiempo y espacio de formación, una etapa en que niños y jóvenes, están siendo preparados para enfrentarse a una sociedad que demandará de ellos valores y principios para convivir en sociedad.

Es claro que este tipo de proposición, evidenciará resultados a un mediano y largo plazo, porque trabaja pensamientos, conocimientos previos, valores y principios, pero podría ser una oportunidad para contemplar una sociedad más responsable y segura.

\section{Antecedentes}

El proyecto de reforma de la "Ley de tránsito para establecer el curso avanzado de seguridad vial" (Salazar y otros, 2004, p. 1) mencionan que en el VII Informe del Estado de la Nación:

\footnotetext{
...El gran rubro de "toda clase de accidentes" tuvo un incremento del 20\% entre los mismos trienios (19982000). En este grupo, los accidentes de tránsito representan la primera causa de muerte accidental. Por sexo, hay una relación de 4,3 varones por cada mujer. En alrededor del $30 \%$ de los fallecidos en accidentes de tránsito, fueron peatones, conductores o acompañantes, se encontraron altos niveles de alcohol en la autopsia...
}

Así mismo explica el proyecto de Ley de reforma, que "los accidentes de tránsito se encuentran entre las 10 primeras causas de muerte en nuestro país (sétima)". "En el año 2001, se registraron 64.802 accidentes de tránsito en nuestro país, murieron 668 personas, más de un 50\% fallecieron en el lugar del accidente (in situ)" (Salazar y otros, 2004, p. 1).

Los diputados (Salazar y otros, 2004, p. 1), explican que "Costa Rica es uno de los países con las más altas estadísticas en muerte por accidentes de tránsito en el mundo", y cita a los autores costarricenses Villasol y Villasol, quienes califican este tema como un "problema social" con una proyección insoslayable", cuando apuntan que:

... no podemos contemplar el tránsito sin la impronta de solidaridad y comprensión que el análisis de cualquier fenómeno humano reclama. Por ello, el científico, el jurista, político o técnico que se ocupe del tránsito debe considerarlo de tal manera, es decir como problema social, entendiendo esta categoría acuñada en las ciencias sociales en su acepción originaria" (Salazar y otros, 2004, p. 1).

Por lo que propone que toda estrategia o plan que se diseñe necesita caracterizarse por mecanismos que provoquen en el ciudadano un despertar del papel que tiene ante la sociedad y que así "decida no saltarse el semáforo, no rayar en curva, decida no tomarse cuatro tragos y coger un volante" (González, 2007), se necesitan estrategias que inciten a los costarricenses a tomar decisiones responsables, con mucho más conocimiento de las consecuencias que pueden tener sus actos

Esta situación requiere de una pronta solución, que no es nueva para los jerarcas del país. En el proyecto de reforma de la ley de tránsito para establecer el curso avanzado de seguridad vial del 2004, se menciona una problemática en la cultura vial del costarricense, cuya causa se le atañe a los insuficientes instrumentos educativos que tiene a la mano el ciudadano. Esto desencadena por lo tanto, una falta de formación y conciencia de la importancia del acto de conducir, además "de una gran corrupción y falta de adecuados controles sobre la destreza y conocimientos elementales del conductor" (Salazar y otros, 2004, p. 2).

Actualmente en el país los estudios que existen, son los que instituciones como las ya mencionadas, muestran en estadísticas, según la revisión de la información encontrada. Por lo tanto se conocen solo números que evidencian un problema, así como algunas causas enumeradas pero no se encuentran explícitas las causas que provocan tal situación ni las posibles 
soluciones. Y esto es lo que ha motivado la propuesta que aquí se presenta.

\section{Ley de tránsito y educación formal}

La ley de tránsito en Costa Rica ha permitido reformas que implican mejoras para una mayor seguridad vial. Actualmente la ley que rige el tránsito es la publicada en el año de 1993.

Esta contempla pautas para los conductores y la circulación de vehículos y aspectos como el seguro obligatorio para los vehículos automotores, las licencias y el permiso de aprendizaje, reglas para la conducción de vehículos, responsabilidad civil, definiciones, regulación del uso de los vehículos del estado costarricense, préstamo institucional de vehículos, disposiciones transitorias, entre otras pautas más específicas. Es decir todo lo referente hacia la parte de información civil. Se incluye además el artículo 67 (Avendaño y Salas, 2003, p. 3) que toma en cuenta la parte formativa de educación vial, exponiendo lo siguiente:

(...) "Para obtener, por primera vez, la licencia de conducir, el solicitante debe cumplir con los siguientes requisitos:

a) Saber leer y escribir. Sin embargo, si el solicitante es analfabeto, podrá obtener su licencia con la previa aprobación de los cursos especiales que establezca la Dirección General de Educación Vial.

b) Aprobar el Curso Básico de Educación Vial, cuyos requisitos se establecerán mediante reglamento, en el cual será obligatorio el estudio de la presente Ley y de otras leyes afines a la materia.

c) Presentar un examen médico que verifique la idoneidad del conductor para el manejo de vehículos o del vehículo específico que se pretende conducir.

ch) Rendir satisfactoriamente un examen práctico para el tipo de licencia a la que se aspira, de conformidad con las disposiciones que para ese efecto establezca la Dirección General de Educación Vial. d) No haber cometido ninguna de las infracciones definidas en el artículo 129 y el inciso k) del artículo 130 de esta Ley, durante los doce meses anteriores a la fecha en la que solicita la licencia por primera vez.

e) Ser mayor de edad, salvo en los casos dispuestos en el artículo 68, para las licencias de clase A, tipos A-1 y A-2...

Es así como la Dirección General de Educación Vial, ofrece a la población un manual oficial, para todo aquel que desee ser conductor y algunos parámetros para el peatón, donde se analizan los aspectos generales de la Ley de Tránsito y los principales factores relacionados con la acción en carretera.

En la presentación del Manual del conductor se explican los tres objetivos para el cual fue creado, el primero referente al campo educativo, el segundo a un cambio de conducta y el tercero a la disminución de accidentes:

1. Formación de mejores conductores y peatones, fomentando un comportamiento de respeto y cortesía entre quienes comparten las carreteras...

2. (...) Lograr un cambio de conducta: el respeto a los dispositivos de seguridad y el cuidado al transitar (...)

3. (...) Disminuir los accidentes (... ) (MOPT, 2007, pp. V-VI).

Los temas que se desarrollan en el Manual son ocho:

1. Aspectos generales del tránsito y la seguridad vial.

2. Legislación del tránsito.

3. Factor ambiental.

4. Factor vehículo.

5. Factor humano.

6. Normas de circulación.

7. Rotondas.

8. El conductor y la contaminación ambiental.

El manual es una guía básica, como se afirma en sus conclusiones con conceptos indispensables para iniciarse como 
conductor, sin embargo explica que "será responsabilidad de cada uno adquirir los conocimientos complementarios necesarios para llegar a ser un buen conductor" (MOPT, 2007, p. 118).

Es claro que esta herramienta de formación que presenta la Dirección General de Educación Vial, se convierte en un complemento de información y formación de los conocimientos básicos que toda persona debe conocer para adquirir su permiso para conducir, pero la formación en valores, que logre extraer de cada ciudadano la responsabilidad de comprender su papel dentro de la sociedad como responsable de su vida y la de los demás, no queda en manos de estas entidades, sino de la entidad encargada de velar por la formación de ciudadanos conscientes de sus deberes y derechos, con una política que promueva la formación de personas capaces de comunicarse de forma asertiva con el mundo, preparados para tomar decisiones efectivas y eficientes como seres independientes y críticos. Practicantes de la solidaridad al compartir como propias las necesidades de los demás, buscando formas de cooperación y concertación entre sectores, asumiendo responsabilidad por la calidad de vida de todos y de las futuras generaciones, sobre la base de un desarrollo sostenible (MEP, 1994, p. 6).

Es decir, el Sistema Educativo debe ser el principal encargado de formar en y para una cultura vial, desde la etapa preescolar y escolar. Con el propósito de que todo ciudadano esté en la capacidad de asumir su función con responsabilidad y respeto.

Existe por lo tanto, la necesidad de contar con una ley que respalde la Educación Vial dentro del Sistema Educativo, como lo expresan Mata ${ }^{1}$ y Sequeira ${ }^{2}$ (2007, Educación vial debe ser constante, parr. 1.), cuando mencionan que:

La población costarricense en edad escolar, así como los sectores formales, no formales e informales, deben tener una educación vial, para que asuman sus responsabilidades, como conductores y peatones.
Así mismo, en el año 2003 en la Asamblea Legislativa, se expone en el proyecto de reforma de los artículos 66, 67 y 129 de la Ley de Tránsito por Vías Terrestres $n^{0} 7331$, la preocupación por:

Una formación vial que inicie desde los primeros niveles de la educación formal y continúe hasta el último año de la secundaria, con programas de estudio que contengan materias permanentes en materia vial y que se complementen con una legislación que responda a las necesidades que existen en este campo (...) (Avendaño y Salas, 2003, p. 2).

Es evidente que la Ley de Tránsito por Vías Públicas y Terrestres no establece dentro de sus artículos la Educación Vial permanente dentro del sistema educativo, por lo que no existe actualmente la obligación o un motivo que respalde la inclusión en los planes de estudio.

\section{Educación Vial y Programas de prevención}

La inseguridad vial que padecen los costarricenses es evidente cuando se observa la gran cantidad de accidentes de tránsito en las carreteras del país. Esto lleva a pensar de forma innegable, que existe un grave problema no sólo social, sino también de salud que nuestra sociedad sufre y que no es una situación reciente, todo lo contrario existe desde ya hace décadas.

Sin embargo, a pesar de las cifras tan alarmantes que se escuchan en todos los medios de comunicación, existe una actitud pasiva ante una problemática que necesita de una pronta solución a corto, mediano o largo plazo.

Las instituciones encargadas de una $\mathrm{u}$ otra manera de la parte de seguridad y educación vial en nuestro país, han hecho esfuerzos por informar y formar a la población en materia vial.

Instituciones como el Consejo de Seguridad Vial (COSEVI), Instituto Nacional de Seguros (INS), Ministerio de Obras Públicas y Transportes (MOPT), 
Ministerio de Educación Pública (MEP), trabajan bajo estrategias de prevención.

Actualmente el COSEVI tiene los siguientes programas:

1. Escuelas seguras.

2. Empresas seguras y temas de seguridad vial.

3. Cursos.

El programa de escuelas seguras es solicitado por las escuelas interesadas. Consiste en llevar a cabo un proceso educativo de promoción de seguridad vial y la prevención de accidentes de tránsito en la escuela o el colegio, con el propósito de disminuir la cantidad de estudiantes lesionados y muertos, así como el fortalecimiento de hábitos seguros en el desempeño del sistema del tránsito.

Con el programa escuelas seguras se espera que cada institución educativa sea un impulsor de la seguridad vial fuera y dentro del ambiente académico en que los estudiantes se desarrollan, para evitar que sufran accidentes. Entre sus objetivos plantean:

“1. (...) Facilitar en los estudiantes la experimentación de situaciones de su rol en el sistema vial.

2. (...) Promocionar estilos de vida seguros en áreas de mayor índice de accidentes de tránsito en el país.

3. (...) Enseñar a los docentes aspectos sobre los factores de riesgo en los estudiantes y poder transmitir los mensajes" (COSEVI, 2004, diapositiva 7).

Uno de los programas que realiza el INS es el de Brigadas de Prevención Infantil en las escuelas, donde miles de niños y cientos de maestros son capacitados por la Institución en materia de accidentes de tránsito, sin embargo, este programa no se desarrolla en todas las escuelas del país, solo las que lo solicitan a la Institución.

Es observable que este tipo de institución, busca formar en la materia a la sociedad costarricense, sin embargo, la información no llega a toda la población del país, inclusive es hasta desconocida. Por lo que lamentablemente son pocos los que logran recibir y capacitarse en este tipo de programas.

En el año 2004 dentro de la Asamblea Legislativa se expuso la preocupación en el proyecto de reforma para establecer un curso avanzado de seguridad vial donde se menciona que

(...) La solución está en proponer mejores métodos preventivos y de educación, para que las personas que aspiran a una licencia de conducir modifiquen radicalmente su percepción de las implicaciones éticas, sociales y de actitud que implica la responsabilidad de colocarse frente a un volante (...) (Salazar y otros, 2004, p. 2).

El Curso avanzado de seguridad vial (CUASEVI) es un curso holístico; participan especialistas en áreas como salud, ciencias sociales, aspectos legales, y así mismo se integran testimonios voluntarios de víctimas sobrevivientes de accidentes, como de familiares.

El propósito de este curso es ser una herramienta para promover una cultura vial, que privilegie la formación desde un punto de vista más humano, para concientizar a los conductores y peatones de su deber de responsabilidad.

Se observa un esfuerzo loable de parte de las instituciones encargadas de erradicar en lo posible esta situación que nos aqueja, con campañas continuas (radiales, vallas, televisivas) sobre el problema de seguridad vial, con el fin de que cada ciudadano construya una cultura que dé certeza a los demás de responsabilidad en sus acciones, sin embargo, hasta la fecha no se han percibido los resultados que se esperan. Accidentes constantes en las carreteras, cargados de negligencia, por conducción temeraria, ebriedad, adelantamientos, no portar licencia, picones, demuestran la ausencia de una formación y cultura vial. 


\section{La educación vial en los programas curriculares}

Jacques Delors en su informe sobre la educación para el siglo XXI, expresa que:

la educación a lo largo de la vida representa para el ser humano una construcción continua de sus conocimientos y aptitudes y de su facultad de juicio y acción. Debe permitirle tomar conciencia de sí mismo y su entorno y desempeñar su función social en el mundo del trabajo y en la vida pública. El saber, "el saber hacer", "el saber ser" y "el saber convivir" en sociedad constituyen los cuatro aspectos, íntimamente enlazados, de una misma realidad (...) (Delors, 1996, p. 110).

Se espera por lo tanto una educación que forme al ser humano para interactuar con su medio de forma responsable para el bien común, al tomar conciencia de sus acciones, mejorará la calidad de vida, fortaleciendo valores que fomenten la armonía en la sociedad.

Así mismo, la Coordinación Educativa y Cultural Centroamericana (CECC) establece cinco fundamentos pedagógicos para la educación del siglo XXI (citado por Argueta, 2002 , p. 8), para guiar hacia una educación centrada en la persona, con el propósito de formar integralmente se presentan:

1. Formación de la persona para mejorar su calidad de vida.

2. Concepción humanística de la educación centrada en valores.

3. La educación integral como política pública para toda la población.

4. Formación basada en el conocimiento científico y su utilidad.

5. El docente como facilitador de la construcción del aprendizaje.

La propuesta educativa para el siglo XXI, desarrolla aptitudes y actitudes que ayudarán al estudiante a vivir en sociedad consciente de sus derechos y obligaciones, que le permitan enfrentar con éxito su participación dentro de la misma.

Es así como para el año 2005, el Ministerio de Educación Pública (MEP) modifica los programas de estudio, estructurados de forma coherente de nivel a nivel, fomentando la transversalidad de los mismos, con el propósito de ser una herramienta que fortalezca valores en y para una formación integral.

Es clara, en los programas de estudio, la necesidad de propiciar mayores niveles de exigencia en la preparación académica y la formación de valores. Se evidencia un compromiso de parte del Estado costarricense de fortalecer y mejorar la educación, con la ideología de que es el camino para forjar "un sistema democrático, equitativo, libre, civilista y solidario", que garantice la calidad de vida para toda la población.

Las palabras expuestas en la presentación de cada programa de estudio, evidencian el interés de presentar y aplicar contenidos y objetivos que respondan ante las necesidades y los retos competitivos de una sociedad globalizada y a la formación de ciudadanos conscientes de sus responsabilidades con el progreso nacional.

El Ministerio de Educación Pública (2005) presenta los programas de estudio, como la columna vertebral del quehacer educativo, que responden a la formación de ciudadanos conscientes de sus deberes y responsabilidades hacia el bienestar nacional.

Con la transversalidad se pretende solventar eficazmente los cambios sociales, económicos, culturales, científicos, ambientales y tecnológicos del siglo XXI. Esto conduce a un cambio radical en el programa curricular, donde no sólo se pretende incorporar nueva información, sino también desarrollar y fortalecer valores, actitudes, habilidades y destrezas para la vida. Sin embargo, dentro de los cuatro temas transversales de este programa no se incluye el tema educación vial.

Actualmente el único programa que contempla la Educación Vial como contenido específico es Estudios Sociales en primer ciclo (Español en el mismo ciclo sólo en el tema de Comunicación), sin embargo, se evidencia una ausencia total (a excepción de cuarto con un tema) en los programas de segundo ciclo y los primeros cuatro años de la educación secundaria. 
En undécimo año se observa dentro del programa de Cívica los siguientes temas: Prevención de accidentes y normas del conductor responsable: prevención de accidentes: manejo preventivo (elementos y actitudes), regulación de licencias y tipos de licencias, señales de tránsito: información, prevención y reglamentación. Además el INA ofrece en algunos colegios la educación vial como tecnología.

Los programas, por lo tanto no contemplan en todos los niveles la formación vial dentro de la educación costarricense, es decir no es una formación continua, no promueve un proceso de formación coherente de nivel a nivel, dejando de lado así, la base pedagógica para una formación integral en una etapa temporal y espacial ideal para que se dé un aprendizaje significante.

La propuesta curricular promueve un concepto de educación integral donde el estudiante pueda lograr desarrollar habilidades, destrezas y sobre todo valores para la vida, enfocados hacia un bienestar individual y social.

Es por esto que desde el 2004, dentro de la Asamblea Legislativa se expone la preocupación de incluirla dentro de los programas curriculares, como un proyecto de reforma a la Ley de Tránsito cuando se menciona: "El Ministerio de Educación Pública, vía adecuación curricular deberá incluir en los planes de educación en cívica de las escuelas y colegios de segunda enseñanza, los contenidos del curso en seguridad vial" (Salazar y otros, 2004, p. 4). Esto con el fin de ser un complemento que refuerce una formación continua en la materia vial.

Sin embargo, las acciones deben ir más allá de un curso en seguridad vial, como lo explica Mata al decir:

(...) el Ministerio de Educación está enfocado en tres estratos a saber: 1) analizar los contenidos de educación vial para poder incluirlos en el currículo del país, la cual debe ser continua desde que el menor ingresa a la educación pre-escolar y concluye la secundaria, 2) la transversalidad, para ello se aplican modelos, prácticas, análisis de valores, como responsabilidad, compromiso, el ser ciudadano y la democracia y 3) concretar un convenio con el Ministerio de Obras Públicas y Transportes para que los cursos teóricos de educación vial, que se imparten para obtener la licencia se incluyan en los planes de estudio de los estudiantes de último año (Mata y Sequeira, 2007, Educación vial debe ser constante, parr. 3).

Es evidente la preocupación que existe sobre la falta de educación vial en nuestra educación y sobre todo que están conscientes de la situación que está enfrentando el país ante una problemática que compete a toda una sociedad, una situación que perjudicará hoy y mañana, sino se considera una formación desde edades tempranas.

Por su parte en otros países como Argentina, las autoridades del Instituto de Seguridad y Educación Vial (ISEV), creen firmemente en el deber del Estado por acercar los conocimientos necesarios hacia cada ciudadano, para que logre actuar de manera segura y responsable al transitar. Esto implica “(...) educar para la vida, la salud y el bienestar general (...)”. Estos son elementos imprescindibles para lograr una cultura vial que se caracterice por una sociedad más justa y democrática (Documento ISEV, 2006, p. 5).

Es por esto que los programas de estudio proveen una herramienta indispensable en la formación ciudadana en este campo. Ver al ser humano, dentro de la educación costarricense como aquel individuo que logra acceder al conocimiento mediante un interés innato por transformar el mundo que le rodea, mediante el currículo como instrumento para formar al ser humano y lograr desarrollar y fortalecer habilidades y valores nos brinda la posibilidad de convivir en una sociedad responsable y segura.

La formación del ser humano desde que nace debe ser integral, los valores y principios que le rodean serán su visión de mundo y de esta dependerá su participación en la sociedad, donde pueda ser el protagonista de lo que existe y logre transformar lo que acontece hacia su bienestar. 
Villarini (1996) habla de un ser humano condicionado por su ambiente sociocultural, pero mediante su accionar puede transformar su relación con dicho ambiente. Se mantiene así una actitud de interacción con el medio para modificar conductas y construir situaciones seguras, según los retos que le exija la sociedad.

Las personas aprenden inmersas en el mundo social, aunque el hecho de aprender pueda considerarse individual, el aprendizaje no es un proceso pasivo, basado en la mera receptividad, por el contrario, es un proceso eminentemente activo donde se aprende para construir.

\section{Causas y consecuencias de la ausencia de formación vial}

"Se ha comprobado que el factor humano es el responsable de la mayoría de los accidentes de tránsito que se dan en Costa Rica, de la totalidad de estos, entre el 57 y el 65\%" (MOPT, 2007, p. 71).

Las causas de los accidentes de tránsito por el factor humano, están bien definidas, entre las más comunes están: exceso de velocidad, ingesta de alcohol, drogas, medicamentos, el irrespeto a los señalamientos, adelantamientos indebidos, no guardar la distancia, malas maniobras, hablar por celular, distracciones y estados de ánimo, entre otros provocan accidentes fatales.

Continuamente se escucha en todos los medios de comunicación la gran cantidad de accidentes de tránsito que diariamente se da en nuestras carreteras. Esto conlleva a su vez un sinnúmero de consecuencias no solo a nivel social, sino también económico y cultural.

Debido a que las personas sobrevivientes a tal fatalidad o bien los familiares de las víctimas deben enfrentar la pérdida de seres queridos o la discapacidad como consecuencia, provoca cambios radicales en el núcleo familiar.
En el Simposio de Educación Preescolar de 1995, María Cecilia Calderón expone que el "constante aumento de los accidentes de tránsito o aquellos derivados de la incorrecta aplicación de normas básicas de prevención, originan en nuestro país cuantiosas pérdidas humanas y económicas, haciendo que este factor se constituya en un mal con características de epidemia social de primer orden" (Calderón, 1995).

Así mismo, en el Proyecto de Reforma de la Ley de Tránsito del año 2004, se habla de los accidentes de tránsito como un problema que se extiende no solo al área social, cuando se menciona que:

El impacto de los accidentes de tránsito no solo constituye un drama humano y de tragedia para muchas familias costarricenses, sino que sus efectos involucran un impacto sobre los servicios de salud hospitalaria del país, extendiéndose sus efectos en los campos social, cultural, tecnológico, económico y ambiental de alta complejidad (Salazar y otros, 2004, p. 1).

Lo anterior nos lleva a reflexionar, cuán grave es la permanencia de esta problemática, cuando diferentes campos se ven afectados por una sola causa: la falta de seguridad vial. Esto evidencia la urgencia de solventar pronto y de forma eficiente y eficaz la situación, disminuyendo el impacto que tiene en la sociedad.

Así mismo en el proyecto de ley que reforma la ley de tránsito, donde se pretende establecer el curso avanzado de seguridad vial, se afirma que “(...) en Costa Rica no existe una educación vial que ponga el énfasis en el cambio cultural y de comportamiento responsable que son los elementos más importantes a la hora de estructurar alguna salida al problema de los accidentes de tránsito (...)" (Salazar y otros, 2004, p. 2).

Es decir, se ha contemplado quizá cursos esporádicos, para informar a la población, pero la parte de formación y una verdadera concientización, que podría buscar un cambio cultural observable a mediano o largo plazo, se ha hecho esperar desde ya hace décadas. Faltan acciones que logren un 
despertar en cada ciudadano, donde logre percibir esta problemática como parte de su responsabilidad y de toda la sociedad.

\section{Una alternativa para la educación vial}

Los entornos-espacios, ya sean naturales o más frecuentemente construidos, actúan a través de sus propias características y estímulos, así lo conceptualizan Cano y Lledó (1997), de acuerdo a las interacciones sociales que en ellos se producen, conforman el comportamiento de las personas. Ambos miran el espacio o entorno no neutral, que posibilita que el ser humano pueda transformarlo para satisfacer sus necesidades, un medio que interactúe con él, donde se construye, reflexiona, se sintetiza y se adjudica juicio crítico de este y de sus componentes, es decir, somos agentes modificadores de nuestro entorno, que debemos trabajar "con él y en él" buscando soluciones para un ambiente en el cual podamos desarrollar de forma plena nuestras capacidades cognitivas, sociales y afectivas.

Es por ello que en el ámbito escolar es necesario que el docente se percate que en el entorno donde el niño se encuentra, se lleva a cabo todo un proceso de aprendizaje que se ve afectado positiva o negativamente por el manejo que se le dé a este, es decir, la etapa de formación de todo niño desde que nace será el reflejo de sus acciones futuras.

Los niños desde que nacen comienzan a explorar todo los que les rodea, pues se ven sumidos dentro de un mundo hasta el momento desconocido y se ven en la necesidad de utilizar sus sentidos, para poder descubrir ese mundo tan complejo. La teoría de Piaget supone que luchamos en forma constante por darle sentido al mundo, empezando por nuestros sentidos (etapa-sensoriomotriz) hasta llegar a un pensamiento abstracto (operacional formal), es decir, el niño inicia su proceso de aprendizaje, experimentando, esto implica desde ver, oír y tocar, hasta saborear y oler.

Por eso es importante que cuando se habla del proceso de aprendizaje es necesario recordar, que no se limita sólo al área cognitiva, sino también a la afectiva y social, ya que se espera que cada ser humano tenga una formación integral, donde no sólo obtenga beneficios de la sociedad en la cual vive, sino también pueda aportar todos los conocimientos que ha adquirido para el bien común.

Montessori creía firmemente en una educación integral, donde se adquieren actitudes y aptitudes que necesitará durante la vida como la cooperación, "la coordinación, la concentración, el orden y la independencia” Montessori (1996, p. 15), cuatro habilidades que sin duda todo niño debe desarrollar y poseer. Esto con el fin de ser capaz de participar en la sociedad con una visión orientada al respeto mutuo y a la calidad de vida, dos factores que lograrán mantener un sano equilibrio.

Una de las frases célebres de Montessori (1996, p. 14) referente al concepto que deberíamos tener de la forma o método (estrategias, técnicas) del proceso de enseñanza-aprendizaje se basa en "una ayuda hasta que la personalidad humana pueda conquistar su independencia”, es decir, la educación es un medio para formar al ser humano en y para la vida, donde sea capaz no solo de enfrentar los retos que se le presenten, sino también que tenga la capacidad para transmitir y formar a otros en los principios y valores que la sociedad requiera.

Es así como la educación continua en y para una cultura que promueva valores y principios de cooperación, seguridad, respeto por la vida, criticidad, iniciativa, cortesía, responsabilidad, empatía y tolerancia, desde la etapa escolar, garantiza una formación integral, en un mundo donde prioriza el egoísmo y el irrespeto a la vida, y se olvida que todo cuanto hacemos afecta a toda la sociedad tarde o temprano, 
donde las consecuencias en la mayoría de los casos, son irreversibles.

Autoridades de países como España, Argentina y Venezuela, creen que parte de la solución a mediano o largo plazo de la problemática vial en las carreteras, requiere una formación en la materia desde edades tempranas, como parte del sistema educativo nacional. El Instituto de Seguridad y Educación Vial de Argentina (ISEV) en su Proyecto de Ley de Educación Nacional: "Hacia una educación de Calidad para una sociedad más justa", pone en la educación ciudadana las esperanzas de solucionar esta problemática y mencionan textualmente:

Creemos que, la Educación Vial es parte integrante de la educación ciudadana, a través de ella se garantiza el uso más apropiado del espacio público, se mejoran las relaciones interpersonales de todos los usuarios en el espacio común, se reconocen los límites legítimos que el derecho impone a la libertad de transitar libremente, en fin, se valora el bien común por encima de los intereses particulares, como una forma de asegurar la vida en democracia (ISEV, 2006, p. 8).

Es evidente que el estado debe responder eficaz y eficientemente ante una necesidad de imperante solución, así lo atribuye el ISEV de Argentina al mantener su posición firme en delegarle al Estado la misión de proveer a la población de las herramientas necesarias para hacerle frente a "lo que amenaza la vida y la integridad física", por causa de la inseguridad en las calles al transitar. Una sociedad que sea democrática, no puede vivir las constantes pérdidas humanas por los accidentes de tránsito, donde los derechos humanos se violentan al no permitir un transitar seguro, donde prevalezca el respeto a la vida. Es así como autoridades del ISEV, le adjudican a la educación vial la garantía de una "democracia que proteja la vida, la salud y libertad de todos los ciudadanos.

Según las afirmaciones anteriores, es imposible hablar de educación vial en un país donde el mismo sistema de educación no contempla dentro de su currículo la formación vial de sus estudiantes, quienes no reciben las herramientas necesarias para actuar como seres humanos responsables de sus actos en materia vial, durante toda sus educación formal.

En Venezuela los accidentes de tránsito constituyen un grave problema de seguridad y salud pública. Donde según datos estadísticos cada año mueren aproximadamente 4 mil personas, 10 mil quedan con algún tipo de lesión física o mental, donde más del 90\% de los accidentes de tránsito se deben a factores humanos: el exceso de velocidad; el consumo de drogas y bebidas; la impericia e imprudencia; el desconocimiento e irrespeto a la ley, reglamento y ordenanzas viales, la ausencia de controles, la falta de vigilancia; impunidad; impaciencia y la agresividad (Comisión Interministerial para la Atención, Prevención y Educación Vial (CIAPEV), 2004). Estos factores de irresponsabilidad según las autoridades de este país se deben a falta de educación vial (agente determinante en la mayoría de los siniestros).

Es así como se da inicio en Venezuela al "Programa Nacional Integral de Educación y Seguridad Vial, cuyo propósito general es el de ofrecer un documento que oriente y facilite la administración de educación y seguridad vial, en los diferentes niveles y modalidades del Sistema Educativo Nacional (...)" (Comisión Interministerial para la Atención, Prevención y Educación Vial, 2004, s.p.).

Estas propuestas plantean una solución y se espera empiece a dar resultados cuantificables y observables en las estadísticas de muertes y accidentes en carretera, donde el protagonista no sea el responsable de la tragedia. Por lo que se plantea no sólo la reflexión y la concientización de la problemática que persiste desde ya hace décadas, sino también acciones que consigan disminuir los accidentes de tránsito, aumentando una formación en educación vial, con un programa curricular que fortalezca la información que se recibe en 
primer ciclo y también en segundo ciclo y su desarrollo sea obligatorio dentro de la educación primaria, esperando que se continúe en el tercer ciclo.

Dentro de la justificación del Proyecto Reforma de la Ley de Tránsito, en el 2003 se menciona que la formación en educación vial desde edades tempranas ayudaría a resolver la problemática vial mencionando que

Este tipo de medida no sólo nos brindaría la seguridad vial que la sociedad civil necesita, sino que a mediano plazo contaríamos con una población con una formación vial sólida.

Sin embargo, es necesario reformar la legislación vigente, para que los procedimientos definidos en la misma, delimiten las probabilidades de que personas anden conduciendo vehículos, sin contar con un período de aprendizaje mínimo en la práctica del manejo (Avendaño y Salas, 2003, p. 2).

El papel indispensable que la educación costarricense debe ejercer en materia de educación vial, es impostergable. Contar con un programa curricular de educación vial, abre las puertas hacia una formación real en el área vial de nuestro país, donde se confronte al estudiante como actual peatón y futuro conductor con la realidad que vive, no como producto de factores y casos aislados, sino como un problema que de no resolverse, le afectará de una u otra manera, pero a su vez que comprenda desde la base de sus principios, que los accidentes de tránsito se pueden prevenir con una actuación ciudadana responsable.

Es clara la urgencia de plantear soluciones para minimizar al máximo las muertes en carretera que despliegan un sin número de consecuencias en la sociedad y sobre todo acciones que demuestran una sociedad cada vez más egocentrista. La problemática que se expone es multicausal, por lo que es evidente que áreas como salud, educación, economía y sociedad general tendrán un papel imprescindible.

Se espera que cada estudiante construya una actitud responsable ante si mismo y los demás. Con un juicio crítico de lo que acontece a su alrededor, cobrando conciencia de que sus acciones afectan positiva o negativamente la sociedad en que vive, por lo que tiene una responsabilidad inherente.

Desarrollar una política donde el ciudadano se comunique activamente con el mundo de manera inteligente, respetando los derechos de los demás y asumiendo eficazmente los deberes como ciudadano costarricense según la ley lo estipule, sin olvidar la necesidad de un compromiso por parte de las autoridades gubernamentales, de mantener el tema de educación vial como prioridad nacional y parte imprescindible en la educación nacional.

La sociedad debe conformarse como un lugar de interacción con el medio y las personas, un lugar donde se aprende a ser y hacer. Donde todos puedan dar y exigir responsabilidad, siendo críticos de la cultura que crean y establecen, una cultura flexible a los cambios que acontecen, de forma tal que se busquen mejoras para el bien común, donde prive una cultura de seguridad y responsabilidad de la participación que cada persona realice.

La situación es muy grave y necesita un cambio en cuanto a la formación que están recibiendo nuestros estudiantes y ciudadanos en todo el país.

El Sistema Educativo debe estar presente en la formación de ciudadanos en materia vial, conscientes de sus acciones y la responsabilidad que ello conlleva y así cada docente cuente con las herramientas que demuestren las secuelas no solo físicas, sino también sicológicas que deja a su paso un accidente de tránsito en el individuo y la familia.

Es necesario tener un absoluto conocimiento sobre las señales de tránsito, su función y reglamentación según la ley, pero así mismo aunado a este esfuerzo, es imprescindible conocer la realidad que acontece día a día, las causas y consecuencias que se generan y cómo estas se pueden prevenir con formación vial. 
Los programas oficiales, deben contemplar la educación vial. Este tema debe ser obligatorio en segundo ciclo y la totalidad de tercer ciclo, ya que niños, adolescentes y jóvenes, en edades entre 10 y 17 años aproximadamente, deben recibir formación como actuales peatones y usuarios de transporte y más aún como futuros conductores.

A pesar de que las autoridades del Ministerio de Educación Pública han manifestado el deseo de que se incluya el tema de Educación Vial como eje transversal en los programas de enseñanza, es necesario considerarlo como contenido específico. La educación vial debe ser un continuo desde preescolar hasta secundaria. Que cada estudiante comprenda la diferencia que existe entre un accidente y la negligencia que provoca tragedias. Que logre atender de forma responsable el consumo de drogas enervantes y sus posibles efectos sobre el peatón y sobre todo el conductor.

Cada ciudadano niño, joven, adulto y adulto mayor deben tener presente que en sus manos tiene un "(...) poder y dominio que si no lo canaliza adecuadamente puede ser causante de consecuencias fatales" (...) (MOPT, 2007, p. 72). Por eso la educación es un arma que le garantiza al país un crecimiento social, económico y cultural, donde todos deben ser formados desde edades tempranas en un continuo durante la vida, con miras hacia una cultura vial que les permita a todos un transitar libre y seguro.

\section{Notas}

1. Alejandrina Mata. Viceministra de Educación.

2. Carlos Sequeira. Presidente Ejecutivo del INA.

\section{Referencias bibliográficas}

Argueta, M. (2002). Ejes transversales en el currículo de la formación inicial de docentes. Cartago, Costa Rica: Impresora Obando.

Avendaño, C. y Salas, J. (2003). Asamblea Legislativa de la República de Costa Rica: Proyecto de Ley: Reforma de los artículos 66, 67 y 129 de la ley de tránsito por vías terrestres $n^{\circ} 7331$, de 13 de abril de 1993. San José, Costa Rica. Extraído el 25 de abril de 2007 de

http://www.asamblea.go.cr/ proyecto/15400/15441.doc

Calderón, M. (1995, Febrero). Educación vial para niños de educación preescolar programa preventivo. En A. L. Chávez, M. de los A. Jiménez, S. M. Chacón y N. García (Eds.), Memoria del Simposio de Educación Preescolar: Educar para la vida (pp. 131-135). San José, CR: Universidad de Costa Rica.

Cano, M. I. y Lledó A. (1997). Espacio, comunicación y aprendizaje. Sevilla: DIANA.

Comisión Interministerial para la Atención, Prevención y Educación Vial [CIAPEV]. (2004). Programa nacional integral de educación y seguridad vial. Venezuela. Extraído el 25 de abril de 2007 de http://www. infocentros.gov.ve/viewusuario/docs/ c6/PROGRAMAvial.doc.

Consejo de Seguridad Vial [COSEVI]. (2004). Programa Centros Educativos Seguros. Diapositiva 7. San José, Costa Rica. Extraído el 26 de junio de 2007 de

http://www.csv.go.cr/educacion/ PROGRAMA.ppt

Delors, J. (Comp.) (1996). La educación a lo largo de la vida. En La educación encierra un tesoro (pp. 107-121). México: UNESCO. 
Documento Instituto de Seguridad y Educación Vial [ISEV]. (2006, Julio). Proyecto de Ley de educación nacional: "Hacia una educación de Calidad para una sociedad más justa”. Buenos Aires. Extraído el 26 de abril de 2007 de http://www.isev. com.ar/uploads/EdVial_Reforma.pdf

González, K. (2007). Asamblea Legislativa. Actualidad Legislativa. Boletín de prensa. Plenario: jueves 22 de febrero. Extraído el 1, mayo, de 2007 de http://www.asamblea.go.cr/actual/ boletin/2007/feb07/22feb07.htm

Mata, A. y Sequeira, C. (2007). Asamblea Legislativa. Actualidad Legislativa. Educación vial debe ser constante. Boletín de prensa. Plenario: miércoles 25 de abril. Extraído el 1, mayo, de 2007 de http://www. asamblea.go.cr/actual/boletin/2007/ abr07/25abr07.htm

Ministerio de Educación Pública de la República de Costa Rica [MEP]. (1994). La Política Educativa hacia el siglo XXI. San José, Costa Rica.

Ministerio de Obras Públicas y Transportes [MOPT]. (2007). Manual del conductor (4ta. ed.). San José, Costa Rica: EUNED.

Montessori, M. (1996). Formación del Hombre. México: Editorial Diana S. A.

Organización Mundial de la Salud [OMS]. (2004, Abril). La OMS trata de sensibilizar sobre la importancia de la seguridad vial en el Día Mundial de la Salud. CONSUMER EROSKI. Extraído el 01 de mayo de 2007 de http://www.consumer.es/web/es/ salud/2004/04/07/98358.php

Salazar, C., Malavassi, F. y Guevara, P. (2004). Asamblea Legislativa de la República de Costa Rica: Proyecto de Ley: Reforma de la Ley de Tránsito para establecer el curso avanzado de seguridad vial. San José, Costa Rica. Extraído el 02, mayo, de 2007 de

$$
\frac{\frac{\text { www.asamblea.go.cr/diputado/ }}{\text { dip_02-06/iniciativas/salazar }}}{\text { ramirez02.htm }}
$$

Villarini, A. (1996). El currículo orientado al desarrollo humano integral. Río Piedras, Puerto Rico: Biblioteca del Pensamiento crítico. 